\title{
Developmental care in the neonatal unit
}

\author{
Nishani Lucas ${ }^{1}$ \\ Sri Lanka Journal of Child Health, 2015; 44(1): 45-52
}

(Key words: Developmental care; neonatal unit)

\section{WHAT IS DEVELOPMENTAL CARE?}

Developmental care, introduced in 1986 by Als et al, is a broad category of interventions that is designed to minimize the stress of the neonatal intensive care unit (NICU) environment. Control of external stimuli (vestibular, auditory, visual, tactile) by noise and light reduction as well as minimal handling, clustering of nursery care activities, and positioning of the preterm baby to provide a sense of containment similar to the intrauterine experience is included under the umbrella of developmental care ${ }^{1}$. The goal of developmental care is to provide a structured care environment which supports, encourages and guides the developmental organization of the premature and/or critically ill infant. It recognizes the physical, psychological and emotional vulnerabilities of premature and/or critically ill infants and their families and is focused on minimizing potential short and long-term complications associated with the hospital experience ${ }^{2}$.

\section{WHY DO WE NEED DEVELOPMENTAL CARE?}

While advances in perinatal care have resulted in decreased mortality rates in preterm neonates, morbidity rates remain high. Preterm infants who are born too soon, leaving the security of the intrauterine environment during this critical period, are challenged with an unfavourable environment in the NICU which would further contribute to their morbidity ${ }^{1}$. The preterm infant's rapidly developing brain is particularly vulnerable to a stressful environment and this has short and long term implications for compromised neurobehavioural development. Developmental care has been shown to decrease length of hospital stay and hospital costs and improve weight gain and time to full enteral feeding, as well as improve neurodevelopmental scores at 9-12 months in two systematic reviews and one meta-analysis ${ }^{2}$.

${ }^{1}$ Consultant Neonatologist, Professorial Unit, De Soysa Hospital for Women, Senior Lecturer, Department of Paediatrics, Faculty of Medicine, University of Colombo, Internationally Board Certified Lactation Consultant (IBCLC)
A multi-centre study conducted on 178 very preterm infants across 25 NICUs in Italy and Boston, Massachusetts, in 2006 - 2007, revealed that infants of NICUs with a higher infant centred care (ICC) score, had better attention and self-regulation, were less excitable, had less hypotonia, were less stressed and had greater physiologic and behavioural stability whereas infants of NICUs with a poor Pain Management (IPM) score, had reduced levels of attention and arousal, were more lethargic and had more non-optimal reflexes ${ }^{3}$. The negative impact of the NICU environment can be manifested in a number of ways by the preterm infant such as increased heart rate, decreased oxygen saturation, poor growth due to increased energy expenditure and prolonged hospital stay ${ }^{1}$.

\section{CONTROL OF EXTERNAL STIMULI}

\section{Light reduction}

Why is light reduction important?

Light reduction is important as this facilitates protected sleep which enhances growth and reduces unlimited light exposure as pupillary constriction is absent until around 32 weeks and light goes in through the thin eyelids even when sleeping ${ }^{4}$.

\section{How can we reduce light in the neonatal unit?}

- When designing a neonatal unit it would be important to have individual lights with dimmers placed over the neonatal cots in contrast to one or two bright lights for the whole unit. However, for units with common bright lights we need to strike a balance between visibility to ensure safety and light reduction for each neonate.

- Blinds / curtains can be used to shade brightly lit windows or doors.

- Incubator covers (Figure 1) should be used for babies less than 32 weeks gestation nursed in incubators. The incubators can be covered / uncovered using the multiple flaps according to the infant and care givers specific needs over the 24 hour period e.g. ward round examinations and cares vs sleeping. They should be changed every week or earlier if soiled to minimise infection. 


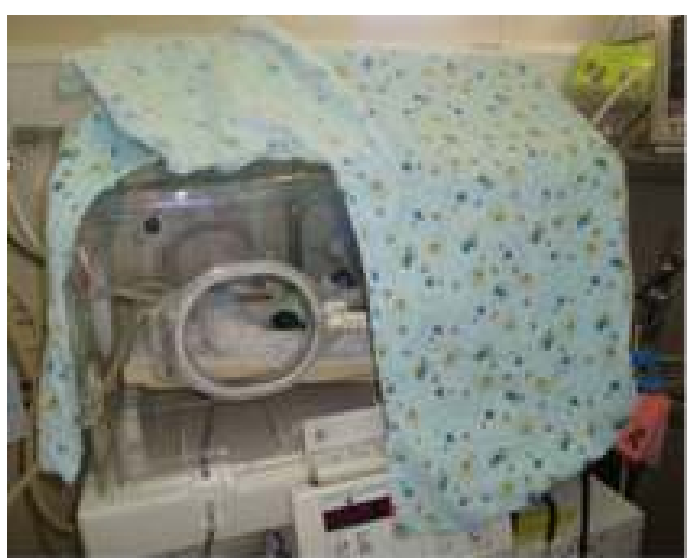

Figure 1: Incubator Cover

Q Copyright Dr Nishani Lacas

- From 32 weeks the baby should be gradually exposed to ambient light during awake periods during skin to skin, feeding or nursing care.

- Cot covers should be used till 34 weeks or till a week prior to discharge.

- Avoid fluctuating bright light on the baby's eyes during care giving procedures. Provide eye protection (cover eyes with nappy/ rolled towel) for babies undergoing cannulation, umbilical line insertion etc. and also during phototherapy.

- Babies who undergo ROP screening should be protected from light for a minimum of 12 hours after the procedure.

- Darkness at night should be provided for infant approaching term by dimming lights near stable babies.

\section{Which babies would benefit from light reduction ${ }^{5}$ ?}

Babies less than 34 weeks gestation and those post Retinopathy of Prematurity (ROP) screening

\section{Visual stimulation of the preterm baby ${ }^{5}$}

- Minimise visual stimuli for babies less than 32 weeks - toys and pictures should not be placed within direct visual space

- Support emerging need for eye contact generally infant shows preference for human faces after 32 weeks.

- Offer opportunities for visual stimulation when the infant is displaying longer attention spans

\section{Enhance development of circadian rhythms ${ }^{6}$}

Dim lights in room at night if safe to do so. A metaanalysis on cycled lighting effects on preterm infants in NICU reports a trend to improved weight gain, shorter length of hospital stay and less incidence of
Retinopathy of Prematurity (ROP) when compared to near darkness or continuous bright light ${ }^{7}$.

\section{Noise reduction}

\section{Why is noise reduction important?}

The fetal response to auditory stimuli (maternal voice) indicated by spontaneous movements is noted as early as 27 weeks of gestation that establishes social attachment and communication during early development. Exposure to noise levels of greater intensities arising from various equipment in the NICU environment act as stressors to the preterm infants ${ }^{7}$. Preterm babies are exposed to nonlanguage high frequency noises like alarms and low frequency noises like infusion pumps, CPAP machines and ventilators along with limited ability to tune out repetitive sounds ${ }^{8}$.

High frequency noises have been shown to cause impaired language development ${ }^{9}$ and $3^{\text {rd }}$ trimester hyperstimulation has been associated with disorganization of the auditory cortex ${ }^{9}$. Excessive auditory stimulation creates negative physiologic responses such as apnoea and fluctuations in heart rate, blood pressure, and oxygen saturation. Preterm infants exposed to prolonged excessive noise are also at increased risk for hearing loss, abnormal brain and sensory development, and speech and language problems. Reducing noise levels in the NICU can improve the physiologic stability of sick neonates and therefore enlarge the potential for infant brain development ${ }^{10}$.

\section{How can we reduce noise in the neonatal unit?}

- Use thick incubator covers

- Talk softly near open cots. Do not talk with the portholes of the incubator open. Avoid loud noise and multiple sound sources.

- Attend to alarms promptly and set alarm volume as low as is clinically safe.

- Decrease volume/tone of telephone ring and no radios in rooms. Audio tapes are not recommended for babies less than 37 weeks.

- Close incubator doors quietly. Do not tap or bang on incubator.

- Discourage the use of the top of the incubator as a writing surface and or storage area.

- Ensure CPAP and ventilator tubing is regularly cleared of $\mathrm{H}_{2} \mathrm{O}$.

\section{Auditory stimulation of the preterm baby}

Encourage parents to talk softly to their baby as cues allow from 28 weeks onwards ${ }^{11}$. Start with soft voice leading on to normal conversation volume/tone. 


\section{Protect babies from noxious odours}

Why it is important?

Taste and smell receptors are thought to be functional and physiologic responses to unpleasant olfactory stimuli have been documented by 27 weeks gestation. Preterm infants will depend on alternate feeding methods like tube or cup feeding with expressed breast milk as they are not able to coordinate sucking, swallowing and breathing. These feeding methods deprive the preterm infant from normal sensory experiences of taste and smell. Further, the unpleasant and noxious odours arising from the hospital disinfectants, solutions, and antibacterial compounds can have a negative impact on already deprived smell and taste sensations. The extended duration of alternative feeding methods also has a negative impact on the sucking behaviour of these preterm infants ${ }^{7}$.

\section{How can we protect babies from noxious odours?}

Open alcohol wipes and antiseptic preparations away from the incubator and infant. Avoid use of strongly scented perfume.

\section{Stimulate baby with pleasant odours}

Parents may familiarize their infant with the smell of breast milk by using milk soaked gauze prior to and during a feed which should be discarded immediately after use.

\section{Minimising oral aversion and suckling on the empty breast}

\section{Why it is important?}

- Oral aversion: Preterm neonates have many negative oral experiences like insertion of endotracheal tubes, suctioning and insertion of orogastric tubes. These negative experiences lead to oral aversion where the neonate rejects any object that comes into contact with the mouth. This may lead to delayed establishment breast feeding ${ }^{13}$.

- $\quad$ Suckling on the empty breast facilitates the sucking behaviour of infants and improves digestion of enteral feeds through secretion of specific digestive enzymes mediated by vagal innervations of oral mucosa. A systematic review on the effect of nonnutritive suckling reports significant decrease in the length of hospital stay and no clinically significant changes in weight gain, energy intake, heart rate, oxygen saturation, intestinal transit time, age at full oral feeds and behavioural state.
How can we minimise the oral aversion and create a positive oral experience?

- Suction orally only when clinically necessary.

- Encourage hand to mouth contact.

- Encourage stable babies to nuzzle at the empty breast during skin to skin contact under close supervision ${ }^{12}$.

\section{Tactile stimulation, minimal handling and clustering nursery activities}

\section{Why it is important?}

The fetus receives rich tactile sensory experiences as it is bathed by the amniotic fluid. During NICU stay the preterm infants are deprived of constant tactile stimulus of amniotic fluid and as well as exposed to varying touch stimulus during medical and nursing procedures. Several studies have made consistent observations on adverse effects of such handling procedures that include hypoxia, bradycardia, sleep disruptions, increased intracranial pressure and behavioural agitation. Therefore appropriate tactile stimulation is indicated at the same time ensuring minimal and gentle handling?

\section{What we can do}

- Swaddling a baby during the non-contact period provides tactile stimulation. It has been shown that swaddled infants have longer sleep and are less aroused; have lower heart rate and are calmer with prolonged sleep and fewer startles; alleviates pain and prevents hypothermia. The study also reported improved neuromuscular development and motor organization following swaddling ${ }^{7}$.

- Cluster cares, but avoid completing a number of potentially distressing interventions at the same time. If an infant indicates signs of stress during handling stop and provide 'time out' for the infant to recoup from that intervention ${ }^{16}$.

- Combine doctors' ward rounds, nurses' care, blood sampling, gases etc. and ensure that the baby has multiple opportunities of undisturbed rest.

- Do not unnecessarily examine stable babies at the end / beginning of each shift

- Gently prepare infant for handling with a soft voice or gentle touch to help promote physiological stability and state organization $^{17}$ i.e. how babies manage to protect their sleep, comfort themselves and organise their sleep and awake states ${ }^{18}$. 
- Interventions should ideally take place when an infant is in a gently aroused state and with consideration of infants' cues. Slow controlled gentle handling, abrupt/fast changes in position are likely to be poorly tolerated for babies under 33 weeks ${ }^{16}$.

- Hold infants during feeding if awake - this includes tube feeding ${ }^{17}$.

- Vary infant head and body positionmindful of infant physiological status and response to handling ${ }^{17}$.

- To soothe infant during uncomfortable procedures contain infant-head and hands in midline, shoulders forward, lower limbs flexed and adducted towards the midline ${ }^{17}$. Elicit help from a parent or another nurse.

- Avoid stimulating the infant with stroking or patting babies under 32 weeks. Patting or stroking may be tolerated for after 32 weeks.

- Where clinically possible consider day/night patterns for interventions from 24 weeks onwards like weigh infant, change bedding and encourage socialization in the daytime to enhance sleep/wake organization.

\section{Positioning ${ }^{17}$}

Before the baby is born he is contained securely by the form boundaries in the uterus by the mother's abdominal musculature, pelvis, spine and diaphragm. The baby's arms and legs are curled up, knees and elbows tucked towards the middle of the body, spine is curved and head is tucked slightly forwards. Maintaining the correct posture is important as muscle tone is still developing until 36 weeks, it helps in ex-utero movement development, prevents postural deformities and helps selfconsoling. In order to ensure the correct posture it is vital to position the baby in such a way with simulated intrauterine boundaries.

\section{The nest}

Supportive positioning technique used should enhance flexion, promote comfort and provide opportunities for movement as well as have simulated intrauterine boundaries. It can be made with rolled up towels / blankets or cot sheets.

*These pictures are the copyright of Dr Nishani Lucas.

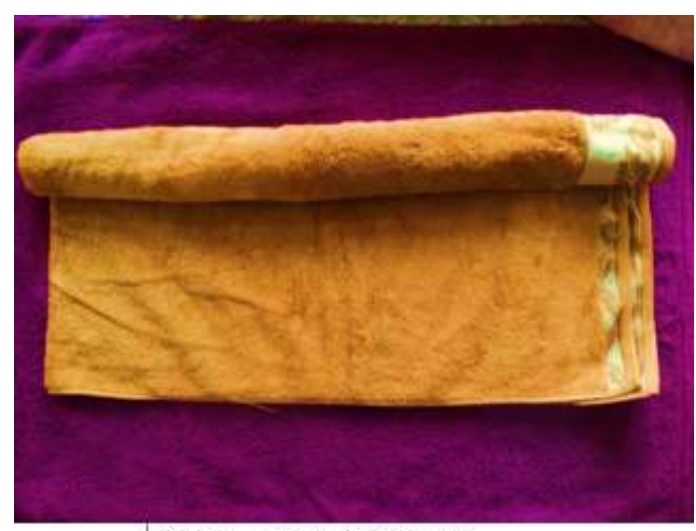

Step 1-Roll the towel

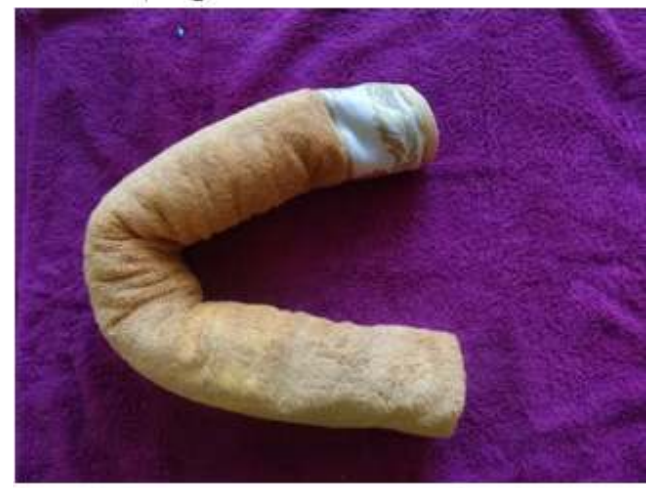

Step 2 - Fold towel into a "U" shape

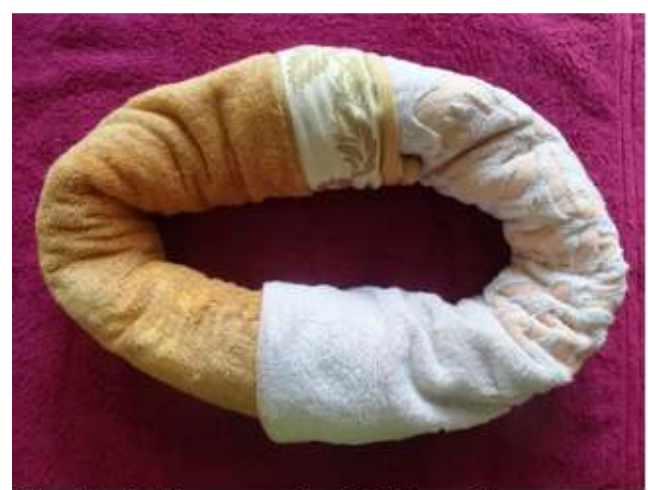

Step 3- Add a second rolled towel to snuggly fit the baby in the flexed position

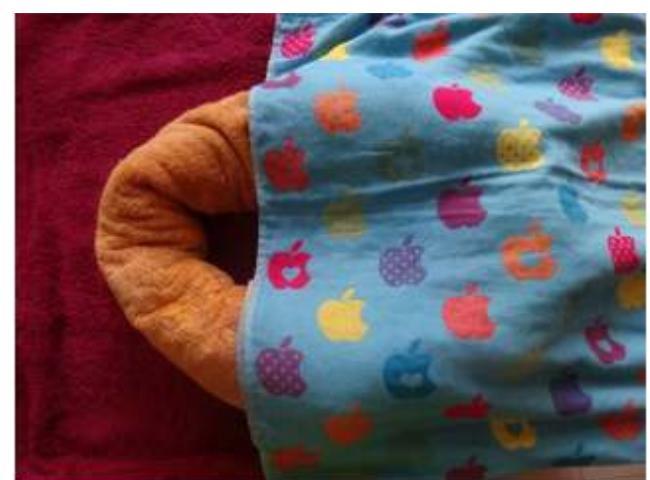

Step 4-Cover with a soft cloth and tuck it in 


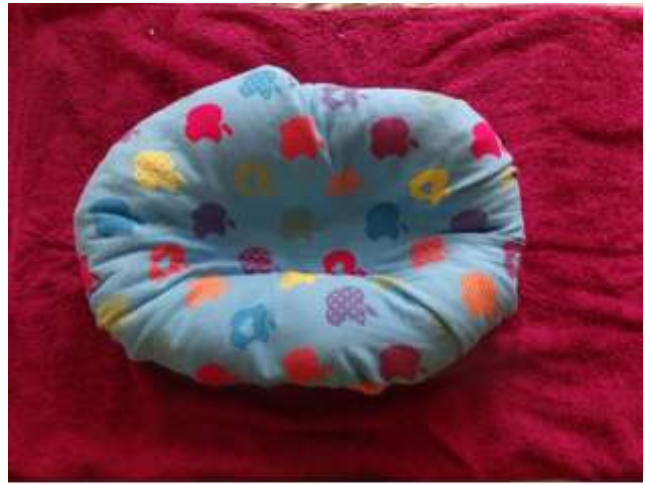

Step 5- The completed nest

\section{Which babies need nests?}

Nests are indicated for babies below 34 weeks, growth restricted but mature babies and acutely ill immobile newborn.

\section{Timing of different positions}

- Prone position is best until the baby is stable.

- Side lying position can be introduced as the baby is becoming more stable and supine position is introduced when preparing to discharge

- Nesting boundaries should be gradually decreased and removed as the baby approaches term / discharge

\section{Cleaning of the nest}

The nest should be cleaned every 48 hours unless visibly soiled and the soft cloth that is used to line the nest should be cleaned daily

Prone position (Figure 2)

In this position the baby must be well supported, as gravity will push the knees out to the sides. It helps breathing movements by supporting the rib cage, reduces reflux, increases time spent in quiet sleep and saves energy and helps faster weight gain.

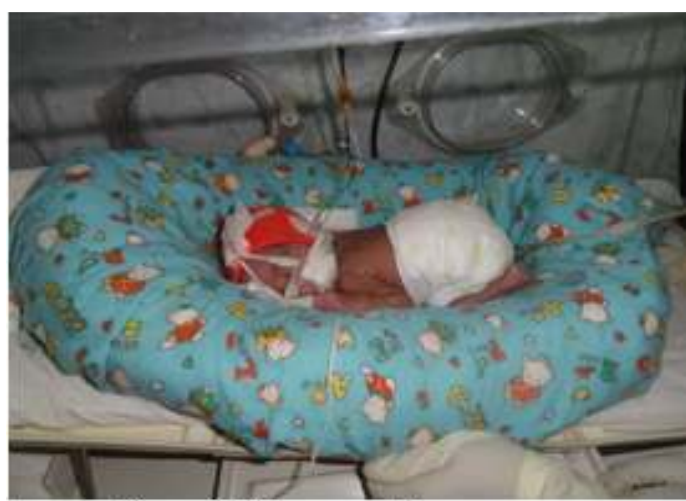

Figure 2: Prone position

(c) Copyright Dr. Nishani Lucas
Kangaroo care is another method of keeping babies in the prone position (Figure 3).

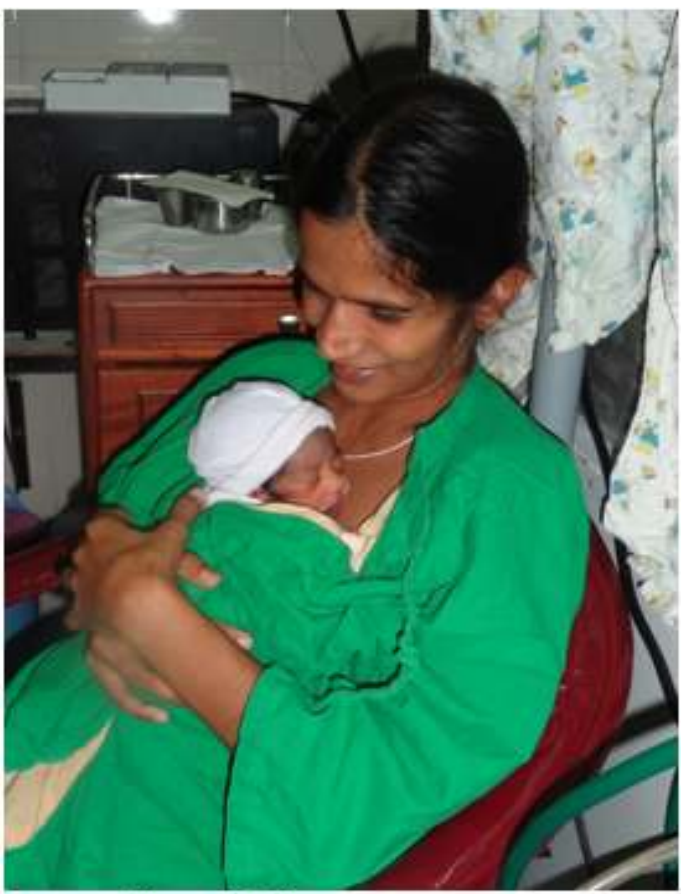

Figure 3: Kangaroo care

(9) Copyright Dr. Nishani Lucas

Side lying (Figure 4)

Helps to get their hands to their mouths for comfort, when upset as part of self-regulation.

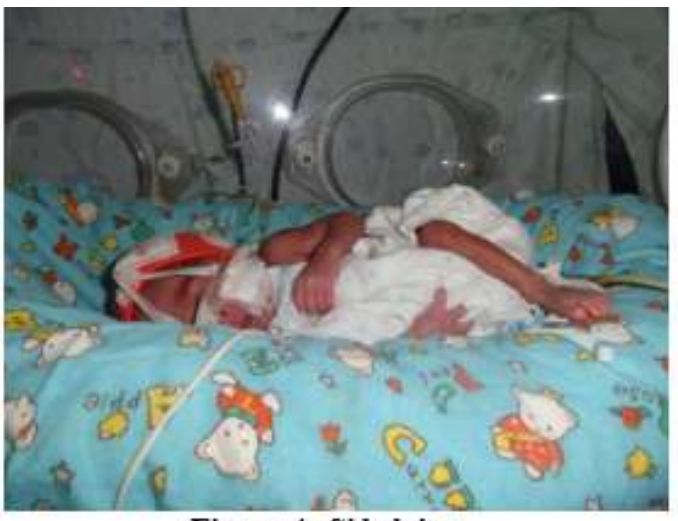

Figure 4: Side lying

\section{(9) Copyright Dr Nishani Lucas}

Babies naturally roll out of this position as shown in Figure 5. Therefore need to use a rolled-up towel to support a tucked up, curled in position. 


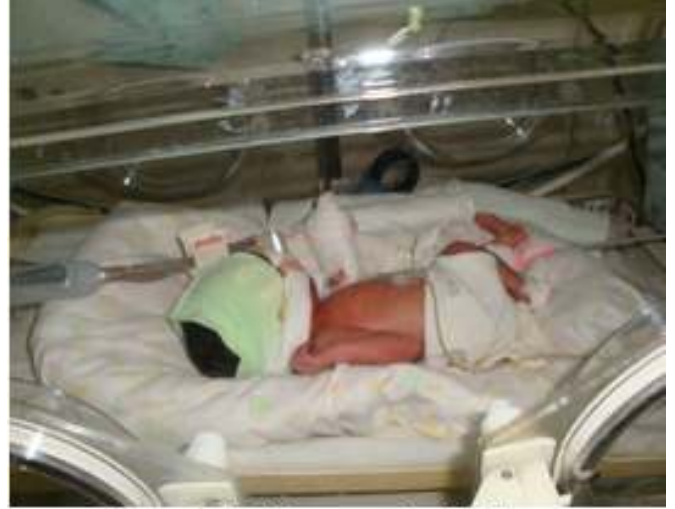

Figure 5: Rolling out of side lying

(c) Copyright Dr Nishani Lucas

Supine position (Figure 6)

Can be introduced during wake periods e.g. nappy changes when the medical condition has stabilized. Face should look up towards the ceiling and should not be turned to either side. Rolled towels / roll pillows can be used to keep head in the midline. This will help the pressure to go down the back of head symmetrically and will prevent premature head shape which will occur if nursed with the cheek on the cot. It will also help the baby to learn to keep the head straight.

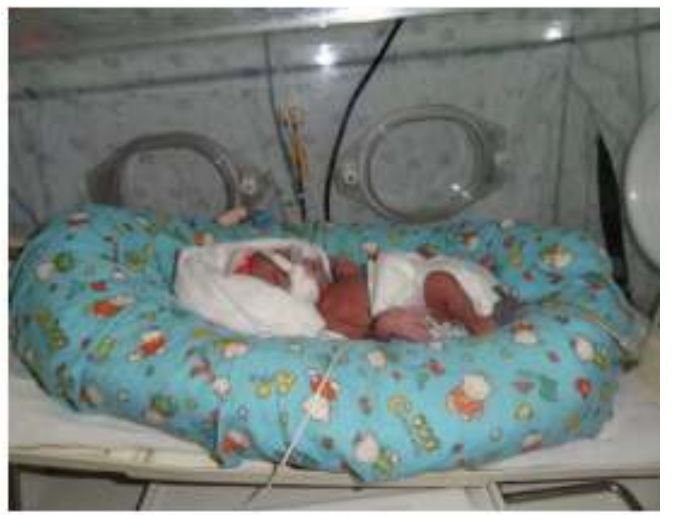

Figure 6: Supine position

(8) Copyright Dr Nishani Lucas

\section{INVOLVE PARENTS IN THE CARE OF PRETERM BABIES}

It is important promote early and continued parental involvement.

- Encourage parents to observe their infants behaviour /cues

- Teach parents to identify infant's readiness for touch and handling and emphasise the infant's potential low tolerance for stimulation.

- Encourage parents to assist with cares where they can, in particular gentle touch, containment during and after handling, top and tail wash and Kangaroo care where appropriate.

- Promote independence and enjoyment of maturing infant by encouraging parents with feeding and cares, in particular containment during and after and Kangaroo care.

\section{UNDERSTAND STRESS SIGNALS OF THE PRETERM BABY AND USING CONSOLING STRATEGIES $^{18}$}

A comfortable baby will have his feet supported (touching the cot / incubator) with a relaxed expression and allow brief eye contact.

The following indicate a stressed baby:

- Arched back

- Frowning

- Toes and fingers spread out

- Thrusting arms and legs in the air

- Yawning / hiccups

- Looking away

- Sudden changes in heart rate or breathing rate

- Suddenly going floppy or stiff

- A scowling face

- Waving arm movements

\section{Consoling strategies}

Containment holding (Figure 7), which entails holding the preterm by placing one hand over the head and the other over the lower back, grasping a finger, suckling on a gauze soaked with breast milk, gentle touch and quiet talking are all consoling strategies that would help the preterm during stressful situations.

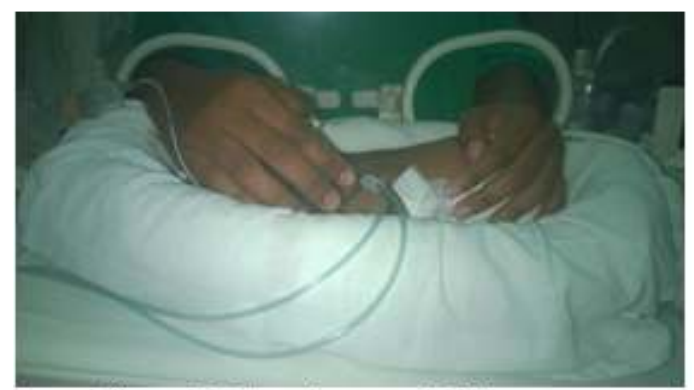

Figure 7: Containment holding

(s) Copyright Dr Nishani Lucas

Implementation of developmental care in the neonatal unit calls for commitment and dedication rather than sophisticated equipment as mentioned above. Therefore the simple measures mentioned above such as reduction of light, noise, noxious odours, oral aversion as well as use of non-nutritive sucking on the empty breast, appropriate positioning 
by use of nests, involving parents in care identifying the stresses of the preterm baby and using appropriate consoling strategies can be easily undertaken in any resource poor setting to improve the neonatal outcomes of our preterm babies.

\section{References}

1. Symington AJ, Pinelli J. Developmental care for promoting development and preventing morbidity in preterm infants. Cochrane Database of Systematic Reviews 2006; CD001814.

2. Coughlin M, Gibbins S and Hoath S. Core measures for developmentally supportive care in neonatal intensive care units: theory, precedence and practice. Journal of Advanced Nursing 2009; 65(10): 2239-48. http://dx.doi.org/10.1111/j.13652648.2009.0 5052.x

3. Montirosso R et al and the Neonatal Adequate Care for Quality of Life (NEO-ACQUA) Study Group. Level of NICU quality of developmental care and neurobehavioural performance in very preterm infants. Pediatrics 2012; 129(5): e1129-e1137. http://dx.doi.org/10.1111/j.13652648.2009.0 $\underline{5052 . x}$

4. Graven SN, Browne JV. Visual development in the human fetus, infant and young child. Newborn and Infant Nursing Reviews 2008; 8(4): 194-201.

http://dx.doi.org/10.1053/j.nainr.2008.10.01 $\underline{1}$

5. Oehler JM. Developmental care of the low birth weight infants. Nursing Clinics of North America, 1993; 28 (2): 289-301.

6. Fielder AR, Moseley MJ. Environmental light and the preterm infant Seminars in Perinatology 2000; 24 (4), 291-8. http://dx.doi.org/10.1053/j.nainr.2008.10.01 1

7. Ramchandran S, Dutta S. Early developmental care interventions of preterm very low birth weight infants. Indian Paediatrics 2013; 50: 765 - 9 . http://dx.doi.org/10.1007/s133120130221-y

8. John Hopkins Continuing Medical Education eNeonatal Review. 2006; 3(6). Available from:

http://www.hopkinscme.edu/ofp/eneonatalre view/Newsletters/0206.html
9. Stromswold \& Sheffield. NICU Noise \& Language Development. Rutgers University Centre for Cognitive Science Technical Report. 2004

10. Brown G. NICU noise and the preterm infant. Neonatal Network 2009; 28(3):165-73. http://dx.doi.org/10.1891/07300832.28.3.165

11. Kenner C, Wright Lott J. Comprehensive Neonatal Nursing 3rd ed. Philadelphia: Saunders; 2003.

12. Turner A, Santangelo S. Developmental and behavioural characteristics of preterm infants. Santa Rosa: NICU INK book. 1999

13. Healow LK, Hugh RS. Oral aversion of the breast fed neonate. Breastfeeding Abstracts 2000; 20(1): 3-4

14. Pinelli J, Symington A. Non-nutritive sucking for promoting physiologic stability and nutrition in preterm infants. Cochrane Database of Systematic Reviews 2005; 4:CD0010 http://dx.doi.org/10.1002/14651858.CD0010 71.pub2

15. Liaw JJ, Yang L, Ti Y, Blackburn ST, Chang YC, Sun LW. Non-nutritive sucking relieves pain for preterm infants during heel stick procedures in Taiwan. Journal of Clinical Nursing 2010; 19(19-20):2741-51. http://dx.doi.org/10.1111/j.13652702.2010.0 $\underline{3300 . x}$

16. Reid T, Freer Y. Developmentally focused nursing care. In: G. Boxwell, editor. Neonatal Intensive Care Nursing. London: Routledge; 2001. p. 14-44.

17. BLISS: The premature baby charity 2005 . Handle me with care - Supporting your premature baby's development. Available from:

http://www.babylink.info/liverpool/parent $\%$ 20information/handle $\% 20 \mathrm{me} \% 20$ with $\% 20 \mathrm{ca}$ re.pdf

18. Hawthorne J. Using the Neonatal Behavioural Assessment Scale to support parent-infant relationships. Infant 2005; 1(6): 213-8.

19. BLISS: The premature baby charity 2005 . Look at me - I am talking to you! Watching and understanding your premature baby. Available from:

http://www.yorkshireneonet.nhs.uk/parents1/look\%20at $\% 20$ me.pdf 